\section{L'Actualité économique}

L'ACTUALITÉ ÉCONOMIQUE

Principles of Economics and the Canadian Economy $\left(2^{\mathrm{e}}\right.$ édition), par R.-C. BELLAN. Un vol., 61/2 po. x 91/2, relié, 556 pages - MCGRAW-HILL COMPANY OF CANADA LTD., 1963

\title{
B. B.
}

Volume 39, numéro 1, avril-juin 1963

URI : https://id.erudit.org/iderudit/1001905ar

DOI : https://doi.org/10.7202/1001905ar

Aller au sommaire du numéro

Éditeur(s)

HEC Montréal

ISSN

0001-771X (imprimé)

1710-3991 (numérique)

Découvrir la revue

Citer ce compte rendu

B., B. (1963). Compte rendu de [Principles of Economics and the Canadian Economy ( $2 \mathrm{e}$ édition), par R.-C. BELLAN. Un vol., $6 \frac{1}{2} 2$ po. $\mathrm{x} 91 \frac{1}{2}$, relié, 556 pages MCGRAW-HILL COMPANY OF CANADA LTD., 1963]. L'Actualité économique,

39(1), 151-151. https://doi.org/10.7202/1001905ar d'utilisation que vous pouvez consulter en ligne.

https://apropos.erudit.org/fr/usagers/politique-dutilisation/ 
ment celle de deux mondes à peu près complètement différents. Seuls les chapitres théoriques fondamentaux sont demeurés intacts. Il y a là dailleurs, nous semble-t-il, rien de moins qu'un paradoxe.

B. B.

Principles of Economics and the Canadian Economy ( $2^{\circ}$ édition), par R.-C. Bellan. Un vol., $6 \frac{1}{2}$ po. x $9 \frac{1}{2}$, relié, 556 pages. McGraw-Hill Company of Canada LTD., 1963.

Cette deuxième édition de l'excellent manuel du professeur Bellan comporte peu de changements notables. Seulement un nouveau chapitre sur l'économie soviétique a été ajouté à l'ouvrage. Ailleurs, certaines explications ont été présentées différemment, quelques graphiques nouveaux ont été inclus, les statistiques ont été mises à jour mais dans l'ensemble l'ouvrage reste celui qui a connu un très grand succès lors de sa parution. Il restera donc très utile aux professeurs et aux étudiants. Pour un compte rendu plus élaboré, on pourra consulter la livraison d'octobre-décembre 1960 de cette revue.

B. B.

Morphologie de la population active à Paris, par Germaine Belleville. Un vol., 6 po. $\times 91 / 2$, broché, 347 pages. - Librairie Armand Colin, 103 Boulevard Saint-Michel, Paris (5e), 1962.

L'étude de Germaine Belleville représente un apport considérable à la connaissance de la répartition de divers groupes sociaux dans le cadre de l'agglomération parisienne. L'auteur présente chacun des quartiers de Paris en indiquant les aspects professionnels et l'état de fortune de la population active qui les habite, ce qui lui permet de dégager des tendances assez surprenantes.

En effet, malgré le brassage provoqué par la guerre et par la crise du logement, il semble que les diverses parties de la ville aient gardé leur aspect traditionnel. Quelques exceptions mises à part, la morphologie professionnelle et sociale de la population a très peu changé et à côté des quartiers dits riches on retrouve les quartiers traditionnellement pauvres ou moins fortunés. Il semble difficile de voir là une sorte de fatalité ou de coïncidence et, selon l'auteur, il s'agit au contraire d'un choix dicté par le souci de garder certaines habitudes.

Germaine Belleville démontre, en somme, que le souci des urbanistes d'opérer un brassage des classes risque de rencontrer des oppositions pratiquement infranchissables, car la population tendra à se regrouper, non pas en fonction des objectifs tels que le décongestionnement des grandes artères et le souci d'une meilleure répartition géographique par. rapport aux professions exercées, mais uniquement en fonction de ses goûts propres.

Optique qui n'est probablement pas aussi valable en Amérique du Nord qu'en Europe, à cause du nombre élevé des agglomérations urbaines totalement nouvelles qui existent sur ce continent, mais qui s'applique dans le cas de Montréal, ou dans celui de Québec.

Les relevés statistiques obtenus par lauteur représentent donc un démenti à des thèses des urbanistes basées sur la logique de l'utilité et il serait intéressant 